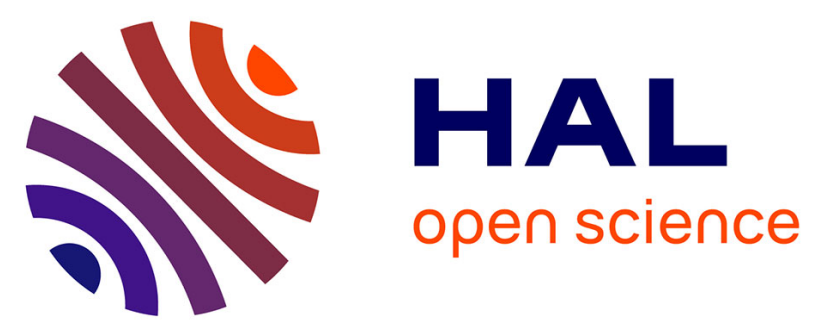

\title{
Movies recommenders systems: automation of the information and evaluation phases in a multi-criteria decision-making process
}

\author{
Michel Plantié, Jacky Montmain, Gérard Dray
}

\section{To cite this version:}

Michel Plantié, Jacky Montmain, Gérard Dray. Movies recommenders systems: automation of the information and evaluation phases in a multi-criteria decision-making process. 16th International Conference on Database and Expert Systems Applications, DEXA'05, 2005, Copenhague, Denmark. hal-00354026

\author{
HAL Id: hal-00354026 \\ https://hal.science/hal-00354026
}

Submitted on 17 Jun 2021

HAL is a multi-disciplinary open access archive for the deposit and dissemination of scientific research documents, whether they are published or not. The documents may come from teaching and research institutions in France or abroad, or from public or private research centers.
L'archive ouverte pluridisciplinaire HAL, est destinée au dépôt et à la diffusion de documents scientifiques de niveau recherche, publiés ou non, émanant des établissements d'enseignement et de recherche français ou étrangers, des laboratoires publics ou privés. 


\title{
Movies Recommenders Systems: Automation of the Information and Evaluation Phases in a Multi-criteria Decision-Making Process
}

\author{
Michel Plantié ${ }^{,}$, Jacky Montmain ${ }^{1,2}$, and Gérard Dray ${ }^{1}$ \\ ${ }^{1}$ LGI2P, Laboratoire de Génie informatique et d'ingénierie de la production, \\ EMA Site EERIE -parc scientifique Georges Besse, 30035 - Nîmes, France \\ ${ }^{2}$ URC EMA-CEA - Site EERIE - parc scientifique Georges Besse, \\ 30035 - Nîmes, France \\ \{Michel.plantie, Jacky.Montmain, Gerard.Dray\}@ema.fr
}

\begin{abstract}
The authors' interest is focused on advanced recommending functionalities proposed by more and more Internet websites w.r.t. the selection of movies, e-business sites, or any e-purchases. These functionalities often rely on the Internet users' opinions and evaluations. A «movie-recommender » application is presented. Recommender websites generally propose an aggregation of the user's evaluations critics according to different relevant criteria w.r.t. the application. The authors propose an Information Processing System (IPS) to collect, process and manage as automatically as possible these opinions or critics to support this multi criteria evaluation for recommendation. The RS (Recommender System) firstly proposes information extraction techniques in order to classify the available users' critics w.r.t. the criteria implied in the evaluation process and to automatically associate numerical scores to these critics. Then multicriteria techniques are introduced to numerically evaluate, compare and rank the competing movies the critics are reported to. Finally the RS is presented as an interactive Decision-Making Support System (DMSS) relying on a sensibility analysis of the movies ranking. A particular attention is paid to the automation of the information phase in the decision-making process: movie comments cartography according to users' evaluation criteria and attribution of a partial score to each critic considered as the expression of a value judgment in natural language.
\end{abstract}

\section{Problematic Introduction}

The impact of Information and Communication Sciences and Technologies is a kernel factor in developing our modes of organization, if not our societies. The economist and Nobel prizewinner H.A.Simon introduced the term of decisional computer sciences [20]. Regardless of how humans are involved in systems nowadays, the systems are so complex that increasingly intricate and inescapable dynamic Information Processing Systems (IPS) are bound to emerge [21]. The aim of such an IPS is to develop models and methodologies that are predominantly compatible with cognitive modes used by human beings when confronted to complex decisions. In particular, in the 
Simon's Human Sciences viewpoint, the different phases of a decision-making process (DMP) don't appear as a linear sequence but as a process with multiple possible cognitive loops. Thus, Intelligence (information), design (representation) and choice (selection) phases are necessarily overlapped in a looped DMP contrarily to the sequential Operational Research viewpoint [22].

The 1990s proved this viewpoint right. Indeed, in the 1990s, there has been an explosion of information technologies, and thus of choices a person faces. Individuals cannot hope to evaluate all available choices by themselves unless the topic of interest is severely constrained. When people have to make a choice without any personal knowledge of the alternatives, a natural course of action is to rely on the experience and opinions of others. We seek recommendations from people who are familiar with the choices we face, who have been helpful in the past, whose perspective we value, or who are recognized experts [24].Today increasing numbers of people are turning to computational Recommender Systems (RS) [4]. Emerging in response to the technological possibilities and human needs created by the World Wide Web, these systems aim to mediate, support, or automate the everyday process of sharing recommendations [24]. Different types of RS are available on the web. We propose to give a brief synthesis in the next paragraph.

During the last few years, RSs have merged to help users in their quest for relevant and personalized information in more and more vast corpus. Several techniques are used to design such systems. The most widespread of them is the "Collaborative Filtering" (CF) [7][10]. The CF deals with the users' preferences w.r.t. the selection of given items (books, movies, etc.). It enables to achieve clusters of users who have expressed similar tastes. To each user, the CF associates a neighbourhood of users who have tastes in common with him. The CF can then propose to a user all the items his neighbourhood has previously appreciated and selected as a recommendation.

A second common approach is the "Content-Based Filtering" (CBF). Applications mostly concerns the selection of documents [14][10]. In that case, the CBF deals with the characteristics, the properties of the documents and propose to the user documents that have similar characteristics or properties to those he has already consulted. The goal is therefore to find semantic neighbouring documents. For several years now, many research studies in the natural language processing (NLP) and the text-mining communities provide relevant tools for this issue [17][6][8][1]. These systems are based on statistic and semantic representations of documents.

RSs have been implemented in several domains on the web. Recommendation of e-business sites has been a privileged application domain [18][19][13]. However users still hesitate to take for granted the RS propositions. Recommendations lack credibility because they are considered as a means of hidden advertising. So a new generation of RS sites has merged: they are based upon the gathering and management of users' feedback experiences. This experience feedback from web users is better accepted in the context of recommendation. Our RS system belongs to this category.

Nevertheless the data gathering and management in RS is often a daunting task because not enough supported. For example, the "expert" user must firstly give his opin 
ion in natural language (NL) and then he is generally compelled to provide with a score (precise values, stars, etc.) consistent with the content of his experience feedback in NL. This score is essential to perform any further numerical evaluation in a recommendation perspective but the consistency checking between it and the NL report isn't supported at all. Furthermore, the evaluation of the compared items (products, services, etc.) generally relies on several criteria and thus necessitates the reiteration of the previous step for each dimension of the evaluation issue! All these «manual » and repetitive tasks have a deterrent effect upon the voluntary "expert" users! We propose to automatically orchestrate these fastidious steps.

As a singular (but representative) case of study, we focus on movie selection support systems, the "movie recommenders" [15][4]: many web sites offer movie advises and evaluations to film lovers looking for a movie on Internet. The NL feedback experience is here the film critic. The critics are picked up either in specialized journals or written down by the RS's users. Scores are associated to the movies' critics. Generally a purely qualitative value is required for this partial score. Imprecise values like stars are also often used, a star representing a bounded interval of scores. Assigning a score to a critic is a delicate and subjective task. However, this redundancy represents a genuine asset in the framework of RS [4]. Indeed, the RS provides its customers with very synthetic pieces of information through these scores: they enable to provide the RS's users with the ranking of the competing movies and any other aggregated indicators such as average scores upon the film buffs' critics. Nevertheless, the webmasters of the RS sites recommend themselves: “... I recommend you to prefer the critic in NL when available rather than to mere synthetic scores..."The critic in NL is a rhetorical element that elucidates and legitimates the afferent score. Indeed, human beings often use reasoning on real numbers (precise or not, reliable or not), thus the critic must be the symbolic transcription of the score. This transcription is a rather tedious and difficult task because subjectivity is inherent to the user's evaluation. For example the meaning of a star can differ from one user to another.

Furthermore, movies are generally evaluated through several criteria (script, actor, cast, production, music...). Thus, a score assigned to a critic is a partial evaluation of the movie w.r.t. a given criterion. The global score attributed to a movie then corresponds to the aggregation of its partial scores w.r.t. each criterion. Until recently, the most common aggregation tool, which is used in multi-criteria decision-making, is the weighted arithmetic mean, as it is the case in many recommender systems [4]. Movies are then ranked according to the aggregated scores they have been assigned. The recommendation principle underlying our RS is based on coupling a base of NL critics and an information fusion system based on multi criteria techniques. All these steps will be succinctly described, but this paper is only focused on the automated transcription procedure that enables to attribute a partial score to a critic in NL and on the critics mapping w.r.t. the set of criteria proposed by the RS.

Section 2 describes the automatic critic/score transcription. Section 3 explains the automated mapping of the critics w.r.t. the set of criteria established by the RS. Section 4 presents the complete processing of the critics in the evaluation, comparison and selection processes implied in the movies RS. 


\section{Critic-Score Transcription}

The first step in our critics processing is how to automatically extract scores from collected movie critics. The score reflects the value judgment expressed in NL in the critic. In this paper we only deal with qualitative scores: the comments and critics will be classified either "positive" or "negative". The automated critic/score transcription consists in the following steps:

- Construction of a movie critics database with the labels "positive" and "negative",

- Extraction of all the lemmatized words from the critics database with the Synapse analyser [23].

- The list of these lemmatized words is the support of our text representation: each critic can thus be represented as a Boolean vector whose each coordinate is a Boolean that expresses the presence or not of the corresponding lemmatized keyword in the critic,

- Classification by supervised machine learning: we have realized a cross-validation campaign to classify the "critics' vectors" in two classes: positive $(\mathrm{P})$ and negative (N). The principle is to build-up several learning sets on already labelled movie critics and then to calculate the associated decision trees who will then be used as classifiers,

- Analysis of the trees' size and their consistency.

Each of these steps is described in the following subsections.

\subsection{Decision Trees}

We use a decision tree classification method. The basic ideas of classification with decision trees are:

- In a decision tree, each node corresponds to an attribute different from the class (in our case a lemmatized keyword of the critic's representation vector) and each arc is associated to a possible value of this attribute (a Boolean here). A tree leaf specifies the expected class for the records described by the attributes path from the root to the leaf.

- In a decision tree, each node should be associated with an attribute different from the class one and that presents the highest informational degree among the attributes that haven't been already considered in the path from the root (this property defines a good decision tree).

- For example, entropy is used to measure the information quantity hold by a node (This notion has been introduced in the Shannon information theory),

In our problematic, the decision tree is build up by machine learning on the P-Nlabelled critics database.

\subsection{A Finalized Representation}

The [1][6][8][17] references present several approaches usable in the documents classification framework. These approaches are focused on representing a knowledge domain and not dedicated to a specific processing of textual information. They are 
intended to be "multiple uses". Here, the philosophy of our modelling method is of different nature, the genericity of the model is left off in favour of the information processing: the aim of the processing is unique, i.e. finding a representation able to optimize critics classification in one of the a priori identified class (here $\mathrm{P}$ or $\mathrm{N}$ class). The idea is then indexing the critics in accordance with the most discriminative lemmatized words for the considered classes. Thus, in our approach the entire critics' corpus representation is guided right from the beginning by the unique processing objective set up by the goal.

\subsection{Knowledge Database}

Our tests have been done on a knowledge database filled up with cinema movie critics coming from a set of Internet websites. In a first experiment, we have asked to a small group of film lovers, so called "the experts" of this experiment, to classify these critics in two different categories: "positive" (P) or "negative" (N). In our implementation, we used 176 cinema movie critics where: 88 critics were labelled as negative, 88 as positive.

\subsection{Complete Key Words Extraction}

A lemmatization with the commercial Synapse analyser is firstly carried out over the whole critics database [23]. Thus, the words coming from the same family but different only on gender or in numeral attribute, are gathered on one unique lemma. Definite and indefinite articles are discarded. The corpus includes of this lemmatized 10765 lemmatized words. A vector representation is associated to the corpus: the higher the frequency of the lemmatized word, the lower its coordinate. Each critic is then represented by a vector in which the $i^{\text {th }}$ coordinate is the frequency in the critic of the $i^{\text {th }}$ most frequent lemmatized word in the learning corpus.

\subsection{Cross Validation Campaign}

We have used the CART method to compute the decision trees [9]. The Figure 1 shows an example of a decision tree used for the classification process. This example illustrates the movie RS domain. The test performed at each node of the tree node tells whether or not a specific word is present in the text for a specific movie critic. To calculate which word should be tested at each node a supervised machine learning procedure is performed: The word associated to a node is computed so as to minimize at this node a measure of the mixing degree of each class. In our case we use the well known "Gini" function described in the CART method [9].

We then proceed to a supervised machine learning. Since the size of the knowledge base (176 critics) is quite small, we have used the cross validation procedure. We have constructed 10 sets of 176 texts. Each of these sets uses 160 texts to compute the decision trees and 16 texts for evaluation. The 160 texts are equally distributed in the two categories, i.e. 80 texts for each category P-N. In the same way the 16 texts are distributed in 8 texts by category P-N. 


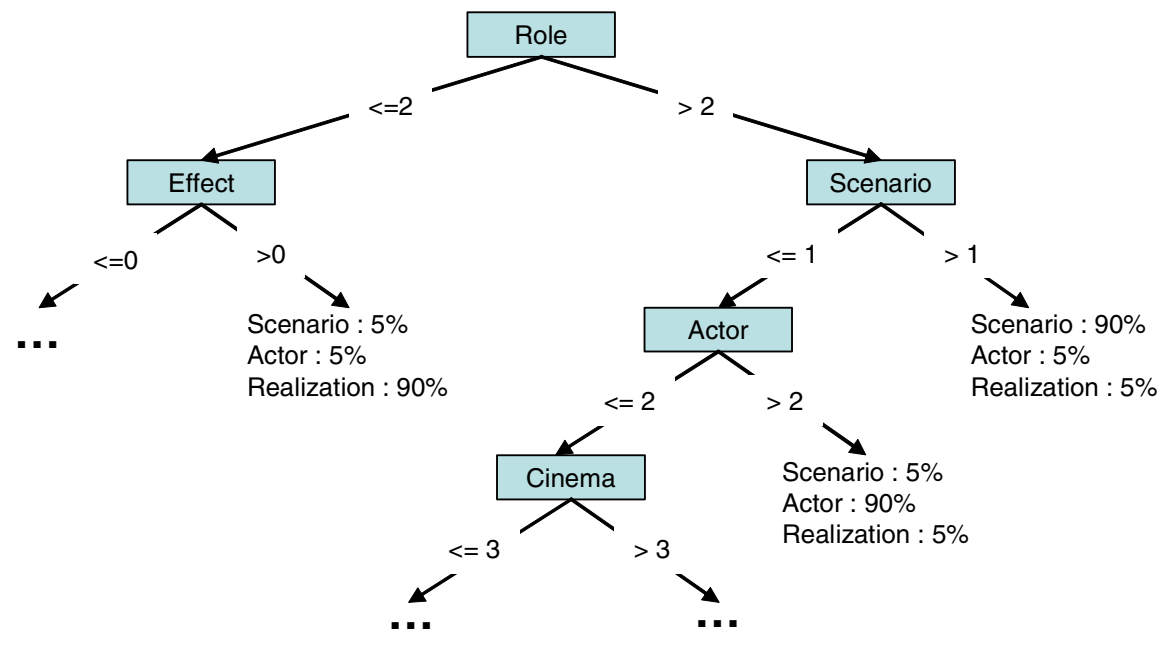

Fig. 1. A Decision Tree Example

\subsection{Performances Estimation Method}

To evaluate the learning results, we use $F_{\beta}^{i}$ score [16]: $F_{\beta}^{i}=\frac{\left(\beta^{2}+1\right) \pi_{i} \rho_{i}}{\beta^{2} \pi_{i}+\rho_{i}}$

$F_{\beta}^{i}$ is founded on precision and recall measures.

In our study $\beta=1$ providing the same weight to precision and recall defined as:

$$
\rho_{i}=\frac{T P i}{T P i+F N i}, \pi_{i}=\frac{T P i}{T P i+F P i}
$$

$T P i, F P i, F N i$, respectively define for a class $i=P, N$ the well classified items ( $i$ classified as $i$ ), the wrong classified items ( $j$ classified as $i$ ) and the omitted items ( $i$ classified as $j$ ). Precision $\pi_{\mathrm{i}}$ for a class $i$ is the proportion of selected items that are correct. Recall $\rho_{\mathrm{i}}$ for a class $i$ is the proportion of target items that were selected.

\subsection{Performances Estimation for Positive/Negative Evaluation Experiment}

Table 1. $F_{\beta}^{i}$ values for the 10 training sets and the $(\mathrm{P})$ and $(\mathrm{N})$ categories

\begin{tabular}{lrrrrrrrrrr}
\hline set & 1 & 2 & 3 & 4 & 5 & 6 & 7 & 8 & 9 & 10 \\
\hline$F_{1}^{P}$ & 0.78 & 0.77 & 0.82 & 0.88 & 0.77 & 0.67 & 0.84 & 0.62 & 0.75 & 0.75 \\
$F_{1}^{N}$ & 0.71 & 0.84 & 0.80 & 0.88 & 0.76 & 0.71 & 0.77 & 0.74 & 0.75 & 0.75 \\
\hline
\end{tabular}

The results are satisfying since the average for the $F_{\beta}^{i}$ values is more than $76 \%$. 


\subsection{Quality of the Calculated Decision Trees}

The cross validation campaign produced ten decision trees. These trees must now be compared in order to verify the homogeneity of the corresponding ten classifiers. In particular, we can verify that the keywords implied in the 10 trees are approximately the same and that the trees structures are similar.

The number of words in a tree goes from 19 at minimum to 31 at maximum. That means that the number of keywords necessary to index the critics in the score transcription application is a drastic and efficient reduction of the initial representation space constituted by the 10765 words of the corpus! Over the set of the 10 computed trees, the total number of words used is less than 80 . The representation space necessary to the classification task is then limited and allows us to plan light calculations processes. In addition, words common to the entire ten trees amount to $20 \%$ of the total number of words in each tree.

Our method allows selecting "a posteriori" the space representation adapted to the intended goal, the most fitted to the problem and the most efficient in compute time. Furthermore this representation is not "purely" statistic: the different paths of the decision tree represent the semantic features of the corpus. The classification process introduces by itself semantic highlights.

These results allow us to use our method as a means to get automatic scoring of opinions expressed in natural language. Although the scores are purely qualitative in this version, we plan to set up 4 categories: "positive", "very positive", "negative" and "very negative". The rejected critics are categorized as neutral.

\section{Criteria Evaluation}

In the last section we have seen that the system is able to automatically attribute a score to a critic written in natural language. In our general presentation of the movie recommenders, we have focused our attention on the fact that the critics are related to $\mathrm{n}$ evaluation criteria (script, actors, image, music, etc.). It is then necessary to adapt the previous transcription "critic to score" for each dimension of the evaluation.

A score relative to one given criterion is said to be a partial score. Extending the automated transcription described in section 2 to partial scores necessitates extracting fragments from the critics that are related to the evaluation criteria. We describe this mapping procedure in the following.

In a second experiment, we have proposed our experts to split up the critics in text fragments w.r.t. the evaluation criteria of the RS. Each fragment is classified in one of the three classes, i.e. the three criteria: "Script" (C1) or "Actor" (C2) or "Movie Direction" (C3). In our implementation, we use 192 fragments from 144 cinema movie critics. 64 fragments are attributed to each criterion.

We have constructed 10 sets of 192 fragments. Each of these sets uses 174 fragments to compute the decision trees, and 18 fragments for evaluation. The 174 fragments are equally distributed in the three criteria, i.e. 58 fragments for each criterion. In the same way the 18 texts are distributed in 6 texts by criterion. 


\subsection{Performances Estimation for Criteria Classification Experiment}

Table 2. $F_{\beta}^{i}$ values for the 10 training sets and three categories

\begin{tabular}{lrrrrrrrrrr}
\hline \multicolumn{1}{c}{ set } & 1 & 2 & 3 & 4 & 5 & 6 & 7 & 8 & 9 & 10 \\
\hline$F_{1}^{C 1}:$ Script & 0.62 & 0.86 & 0.80 & 0.83 & 0.73 & 0.83 & 0.62 & 0.55 & 0.55 & 0.75 \\
$F_{1}^{C 2}:$ actor & 0.3 & 0.92 & 1.00 & 0.83 & 0.83 & 0.91 & 0.83 & 0.91 & 0.91 & 0.74 \\
$F_{1}^{C 3}:$ movie Direction & 0.73 & 0.67 & 0.86 & 0.67 & 0.42 & 0.77 & 0.36 & 0.57 & 0.57 & 0.64
\end{tabular}

The results are quite satisfying since the average for the $F_{\beta}^{i}$ values is more than $73 \%$.

\subsection{Homogeneity for Criteria Classification Experiment}

The number of words in a tree goes from 32 at minimum to 41 at maximum. This representation reduction is rather drastic compared to the 5314 words of the corpus of this second experiment. The number of common words in all the trees is 12: it represents $29,3 \%$ up to $37,5 \%$ of the total number of words in one tree. The total number of words used in the ten trees is only 82. As stated in the first experiment, the representation space necessary to the classification task is quite limited and relevant. Each path from the top to a leaf of the tree represents a multi-word co-occurrence [6]. This notion of co-occurrence is to be related to semantic features of documents representation. There's something more than statistics in the text analysis.

\section{The Recommendation System}

We give in the following a very short description of the complete movie RS as described in [4]. The main goal of this section is to show the interest and role of the previous calculations in the complete RS processing. Sections 2 and 3 have automated the mapping of critics w.r.t. a set of criteria and the association of partial scores to them. These partial scores are then used in a multi criteria quantitative evaluation of movies. Multi criteria aggregation (MCA) is the basis of movies evaluation and ranking in RSs. However, aggregation in RSs is usually reduced to its simplest form: average ratio or for the better weighted average ratio. We have proposed aggregation operators with richer semantics allowing to model different aggregation strategies for evaluation [5]. In this paper and for the sake of understanding, we have made up our mind to carry on the presentation with the weighed average operator as an illustration of the multi criteria analysis in the RS. The global score of a movie is thus a weighted average (WA) of the partial scores obtained for each evaluation criterion. The weights define the decisional strategy but the way they are determined is not discussed in this paper. Furthermore, several critics are available w.r.t. a given couple (movie, criterion). It means that a first aggregation has to be processed to aggregate the partial scores w.r.t. this couple. A simple arithmetic average is considered in this paper for this first aggregation level. A grid evaluation (figure 1) of the movies candidates 
according to a set of criteria is at the origin of the decision support functionalities. The basic idea of movies evaluation refers to collective choice theory, identifying the criteria as voters whose votes are the critics (the value judgments they convey), and the movies as candidates for election. The scores embodied in the critics and assigned in the grid allow the differing intensities of voter preferences to be taken into account: each vote is an evaluation of a movie $i$ with respect to a criterion $j$. The overall score of a movie corresponds to the aggregate of partial preferences obtained for each criterion, and can thus be equated with a criteria-driven election procedure.

The score associated to the critic corresponds to a degree of satisfaction that is converted into a color code ranging from non-satisfaction (red) to complete satisfaction (green), and which may take any intermediate value (depending on the granularity of the classifier described in section 2).

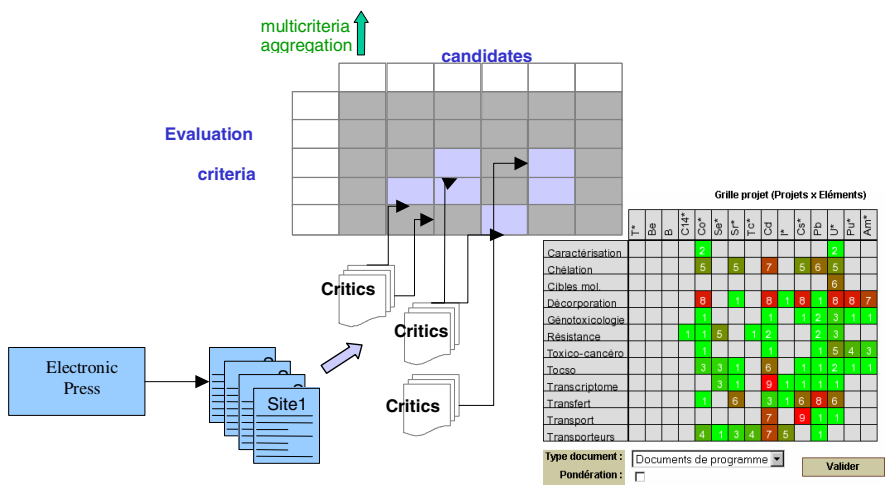

Fig. 2. Multicriteria evaluation for movie critics

The evaluation process for the film lovers group is showed in Fig. 2. As soon as a critic is put in the knowledge base, it is first automatically mapped on the evaluation grid in a criteria line for a film column with the classification technique described in section 3. A score is then automatically attributed to the mapped critic through the transcription critic/score in section 2 . Then the average scoring is computed in the cell where the critic has been mapped. The movie evaluation corresponding to the critic is automatically computed with the WA operator. $W A\left(X_{k}\right)=\sum_{j=1}^{n} \lambda_{j} x_{k}^{j}$ where WA is the weighed average, $\lambda_{j}$ the weight for criterion $j, x_{k}^{j}$ the score for movie $k$ w.r.t. criterion $j$ and $n$ is the number of criteria.

\subsection{Argumentation}

For the considered aggregation operator, the best movie, noted $X_{1}$ is then obtained. By definition we have: $\forall i, W A\left(X_{1}\right) \geq W A\left(X_{i}\right)$. We have shown in [12] how it's possible to automatically extract the most outstanding critics from the knowledge base, i.e. the main discursive items that explain this ranking. The calculation details are given in [2][3] for more complex operators than WA ones. The basic idea of the algorithms 
is to rewrite any mathematical entity necessary to the argumentation function as a ranked sum of criterion rating contributions to this mathematical entity (it corresponds to an interpretation of the relative importance of criteria in terms of marginal contributions). For example, in the case of the WA operator, the reasons for which movie $X_{1}$ is preferred to movie $X_{i}$, is got by rewriting formula $W A\left(X_{1}\right)-W A\left(X_{i}\right)$ as: $W A\left(X_{1}\right)-W A\left(X_{i}\right)=\sum_{j=1}^{n} \lambda_{j} \cdot\left(x_{1}^{j}-x_{i}^{j}\right)=\sum_{j=1}^{n}$ Contribution $($ criterion $j)$.

Then, the most significant contributions are selected through order of magnitude reasoning. It provides the most determinant criteria $\left\{j^{*}\right\}$ in the choice of $X_{1}$ rather than $X_{i}$ : justification is the basis of recommendation [3]. When the criteria $\left\{j^{*}\right\}$ have been identified, the RS can then select the bets critics in the grid cells (1, $\left.\left\{j^{*}\right\}\right)$ and the worst ones in the grid cells (i, $\left.\left\{j^{*}\right\}\right)$ in the knowledge base to elucidate the choice in natural language for the RS user.

\subsection{Reliability of the Classification}

Now consider the reliability of the classification for the film lover and customer of the RS. Let's write $\mathbf{x}_{i}$ the vector of the partial scores obtained by movie $X_{i}$.

To define the decisional risk, i.e. the reliability of the recommendation, we propose to measure it according to a distance notion between movie $X_{1}$ and movie $X_{i}$, founded on the $\mathrm{L}_{1}$ norm and including the concept of improvement effort [4]:

$d\left(\mathbf{x}_{1}, \mathbf{x}_{i}\right)=\min \left\|\vec{\delta}_{1}^{i}\right\|_{1} / W A\left(\mathbf{x}_{i}+\vec{\delta}_{1}^{i}\right)=W A\left(\mathbf{x}_{1}\right)^{1}$

The risk notion introduced is quite different of a conventional probabilistic one and is more in line with a sensitivity analysis of the movies ranking to any external information disturbance. Thus on the basis of this distance, we define the decisional risk notion as the expression $\boldsymbol{r}$ associated with the movie classification stability defined as follows: $r=1-\min _{\substack{i=1 . . p \\ i \neq 1}} \frac{d\left(\mathbf{x}_{1}, \mathbf{x}_{i}\right)}{n}$ where $\mathrm{p}$ is the number of competing movies.

Thus, the lower the distance between $X_{1}$ and its challengers, the more sensitive the ranking and the less reliable the selection of $X_{1}$ : indeed a "small quantity of information" (i.e. few but relevant critics) could be sufficient to modify the movies ranking. We consider that we are able to make a decision when the risk is going under a fixed threshold $C_{r}$. This threshold defines the decision acceptability in this scheme. The calculus of $d\left(\mathbf{x}_{1}, \mathbf{x}_{i}\right)$ gives the least effort to provide (in the sense of norm L1) in order that the evaluation of $X_{i}$ should be at least equal to the one of $X_{1}$. This calculus enables to determine the criteria on which $X_{i}$ should (be) improve(d) first in order to improve as much as possible its overall score. In other words the expression $d\left(\mathbf{x}_{1}, \mathbf{x}_{i}\right)$ provides the criteria where $X_{i}$ must necessarily progress to reach $X_{1}$ with a minimal effort. Thus, the RS provides the film lovers with the sensitive or critical dimensions

\footnotetext{
${ }^{1}$ When the aggregation operator is a WA this optimization problem is a mere simplex.
} 
of the evaluations. The RS manager can also use this kind of information to relevantly complete his knowledge base with corresponding additional critics [5].

Through the concept of risk and acceptability of the decision, we have defined a reaction feedback loop upon the content of the critics knowledge base used for the movies evaluation. Pointing out only the most relevant movies and criteria for which additional critics should be of great interest modifies the selection process dynamics. The management of the RS is thus represented as a control loop: the risk accompanying the decision is the controlled variable and is strongly linked to the entropy of the knowledge base managed by the RS. Each of the three phases-Intelligence, Design, Choice — of the decision-making process is identified to a function of the control loop: actuator, process and regulator. This viewpoint thus proposes a way the iterative cognitive phases-Intelligence, Design and Choice-which represent the Simon's Human Sciences vision of the decision process, can be represented in a control theory framework. The cognitive loop of the Simon's model of DMP, we evocated in the introduction, is seen as a control loop on the decisional risk, itself related to the entropy of the critics corpus.

\section{Conclusion}

We have developed a Decision Making Support System combining both text-mining techniques for the information phase and multi criteria analysis techniques for the justification and selection phases of movies in a RS. This movies RS approach supports the idea of cognitive automation of collective decision-making process: the Simon's cognitive and descriptive model of decision-making process is here interpreted in a cybernetic framework. Our RS is a synthetic tool to automate or to strongly support the information, evaluation, comparison, and selection steps in the web-recommenders problematic. This paper is focused on the automatic transcription from critics into scores. This step associated with an automatic critics mapping procedure produce a complete automation of the information phase implied in the collective DMP implied in the RS. In this kind of DMP (Simon's IPS model), the information phase plays a major role and that's why its automation is an essential step in the RS. Exhaustive cognitive automation, as proposed in this paper, is probably (and hopefully!) a utopian aim but should represent one ideal for the Computer Science and Process Control Theory communities in the era of numerical networks. Indeed knowledge acquisition and processing in always greater corpus are probably a more crucial problem to achieve the «righteous » decision than the search of an apparently optimal decision that relies on many modeling hypotheses and simplifications. That's what Simon pointed out in 1947[21]!

\section{References}

1. Abramowicz, Witold and Piskorski, Jakub, (2003). Information Extraction from Free-Text Business Documents, Effective Databases for Text \& Document Management, p 12-23, IRM Press, Hershey, USA.

2. Akharraz, A., Montmain, J., Mauris, G (2002a). A project decision support system based on an elucidative fusion system. Fusion 2002, Annapolis, Maryland, USA. 
3. Akharraz, A., Montmain, J., Mauris, G (2002b). Fonctionnalités explicatives d'un système de fusion de connaissances collectives par intégrale de Choquet 2-additive. LFA'2002, Montpellier, France.

4. Akharraz, A., Montmain, J., Mauris, G (2004). Elucidation and risk expressions of a movie recommendation based on a multi-criteria aggregation with a Choquet integral. IPMU 2004, 10th International Conference on Information processing and Management of uncertainty in Knowledge-Based Systems, Genova, Italy..

5. Akharraz, A., Montmain, J., Mauris, G. (2004). A cybernetic modeling of a multi-criteria decision process in organization. EURO XX, 20th European Conference on Operational Research, Rhodes, Greece.

6. Besançon, R. and M. Rajman (2000). Le modèle DSIR : une approche à base de sémantique distributionnelle pour la recherche documentaire. Revue TAL’2000, n $41(2)$, pp. 1-27

7. Breese, J.S., Heckerman, D., Kadie, C., Empirical analysis of predictive algorithms for collaborative filtering. Proceedings of the 14th Conference on Uncertainty in Artificial Intelligence, Madison, USA, pp. 43-52.

8. Chauché, J. (1990). Détermination sémantique en analyse structurelle : une expérience basée sur une définition de distance. TA Information, 31(1), pp 17-24.

9. (Crawford et al., 1991) Crawford, S., Fung, R, Appelbaum, L., Tong, R. (1991). Classification trees for information retrieval, Actes $d u 8^{\text {th }}$ International workshop Machine Learning, Northwestern university, Illinois.

10. Herlocker, J.L., Konstan, J.A., Borchers, A., Riedl, J. An algorithmic framework for performing collaborative filtering. Proceedings of the Conf. on Research and Development in Information Retrieval, Berkeley, California, USA.

11. Lang K. (1995). NewsWeeder: Learning to filter netnews. Machine Learning. Proceedings of the Twelfth International Conference, Lake Tahoe, California.

12. Montmain, J., Akharraz, A. and Mauris, G. (2002). Knowledge management as a support for collective decision-making and argumentation processes. IPMU'2002, Annecy, France.

13. Montmain, J., Denguir, A., Mauris, G. (2005). How deriving benefits from expert advices to make the right choice in multi-criteria decisions based on the Choquet integral? European workshop on the Use of Expert Judgement in Decision-Making, Aix-en-Provence, France.

14. Pazzani, M., Muramatsu, J., Billsus, D., Identifying interesting web sites. Proceedings of the Thirteenth National Conference on Artificial Intelligence, Portland, Oregon.

15. Perny, P., Zucker, J-D. (2001). Preference based search and machine learning for collaborative filering : the "film-conseil" movie recommender system. Information, Interaction, Intelligence, 1(1), pp.9-48.

16. Van Rijsbergen, C.J. (1979). Information Retriaval, Butterworths, London 1979, $2^{\circ}$ edition. http://www.dcs.gla.ac.uk/Keith/Preface.html.

17. Salton G. The SMART Retrieval System - Experiments in Automatic Document Processing », Prentice Hall, Englewood Cliffs, NJ,1971.

18. Schafer J.B., Konstan J., Riedl J. (1999). Recommender Systems in E-Commerce. Proceedings of the ACM'1999 Conference on Electronic Commerce, Denver,Colorado, USA.

19. Schafer, J.B., Konstan, J., Riedl, J. (2001). E-Commerce Recommendation Applications. Journal of Data Mining and Knowledge Discovery, Vol. 5 Nos. 1/2, pp. 115-152.

20. Simon, H.A. (1977). The new science of management decision. Prentice Hall, New Jersey.

21. Simon, H. A. (1983). Administration et processus de décision. (trad. (Simon, 1947). Économica, Paris.

22. Simon, H.A. (1997). Models of bounded rationality. MIT Press, Cambridge Massachusetts.

23. Synapse analyzer : http://www.synapse-fr.com

24. Terveen, L., Hill, W. (2001). Beyond recommender systems : helping people help each other. In HCI in the milenium, J. Caroll, ed., Addison-Wesley, pp. 1-21, 2001. 